\title{
MONITOREO DE LA CARGA FÚNGICA AMBIENTAL Y DE OTROS BIOAEROSOLES EN UN DEPÓSITO DE RESTOS MOMIFICADOS DEL NOA del Museo de la Plata (Argentina): un estudio de caso
}

\author{
DANIELA S. NITIU1,4, ANDREA C. MALLO',5, LORENA A. ELÍADES ${ }^{2,4}$, MARIO N. \\ SAPARRAT ${ }^{3,4}$ y HECTOR R. VAZQUEZ ${ }^{6}$
}

\begin{abstract}
Summary: Monitoring of the environmental fungal load and other bioaerosols in a deposit of mummified remains of the NOA of the Museum of la Plata (Argentina): a case study. The aim of this work was to carry out a survey on the airborne fungal charge and other bioaerosols associated with a repository of mummified legacy from the Argentinian North West from the La Plata Museum. Two types of monitoring were performed: i) a viable and non viable aerobiological sampling in the exterior air of the repository, in the indoor air within the same and inside 3 cabinets containing human remains; and ii) a study and culture of the micobiota associated to the surface of materials with evident signs of biodeterioration. Based on the aerobiological sampling, the greatest diversity and spore concentration was registered in the outside environment, being Cladosporium cladosporioides, Alternaria sp. and Penicillium sp. the most representative taxa. Either inside the repository of the 3 cabinets, showed a particular fungal charge, the presence of Penicillium sp., Cladosporium sp. and Aspergillus sp. was characteristic. The analysis of contact samples makes evident the presence of Cladosporium herbarum, Penicillium frequentans and Talaromyces helicus. The knowledge of fungal microorganisms present in indoor environments is essential for heritage conservation and human health subjects.
\end{abstract}

Key words: Fungi, indoor environment, cultural heritage, non viable - viable system, conservation, biodeterioration, museum.

Resumen: El objetivo del trabajo fue llevar a cabo un relevamiento de la carga fúngica ambiental y otros bioaerosoles asociados a un depósito de restos momificados del NOA del Museo de La Plata. Se realizaron dos tipos de monitoreos, i) uno del tipo aerobiológico (viable y no viable) en el aire exterior del depósito, en el aire interior del mismo y en el interior de 3 de sus vitrinas donde se hallan restos humanos; y ii) otro que consistió en el estudio y cultivo de la micobiota asociada a la superficie de materiales con signos evidentes de deterioro. En base al muestreo aerobiológico, se registró la mayor diversidad y concentración esporal en el ambiente exterior siendo Alternaria sp., Cladosporium cladosporioides y Penicillium sp. los taxa más representativos. Aunque el interior del depósito como de cada vitrina analizada mostró una carga fúngica particular, la abundancia de las esporas correspondientes a Aspergillus sp., Cladosporium sp. y Penicillium sp. fue característica. El análisis de las muestras de contacto evidenció la presencia de Cladosporium herbarum, Penicillium frequentans y Talaromyces helicus en asociación a las momias analizadas. El conocimiento de los hongos presentes en ambientes interiores y sobre materiales de valor patrimonial constituye un elemento clave para el establecimiento de pautas de control para su conservación.

Palabras clave: Hongos, ambiente interior, patrimonio cultural, sistema no viable - viable, conservación, biodeterioro, museo.

\footnotetext{
${ }^{1}$ Cátedra de Palinología. Facultad de Ciencias Naturales y Museo, Universidad Nacional de La Plata. Calle 64 N³. 1900.

La Plata. Argentina. E-mail danielanitiu@yahoo.com.ar

${ }^{2}$ Instituto de Botánica Carlos Spegazzini, UNLP

${ }^{3}$ Instituto de Fisiología Vegetal UNLP.

${ }^{4}$ CONICET

${ }^{5} \mathrm{CIC}$

${ }^{6}$ Unidad de Conservación y Exhibición del Museo de Ciencias Naturales de La Plata.
} 


\section{INTRODUCCIÓN}

Uno de los pilares fundamentales de los museos es la preservación del patrimonio cultural e histórico, el cual consiste en un conjunto de bienes culturales definidos por la Convención de la UNESCO (1970) como aquellos objetos que, por razones religiosas o profanas, hayan sido expresamente designados por cada estado como de importancia para la arqueología, la prehistoria, la historia, la literatura, el arte o la ciencia los cuales deben ser protegidos y resguardados junto con sus debidos registros en sitios acordes que garanticen la integridad del mismo. Los Museos deben tener aseguradas la guardia y el control de su manejo, puesto que esto tiene asociado el cumplimiento de legislaciones que avalan su cuidado y la tutela de los materiales culturales que albergan (Harvey, 1977).

Los problemas de conservación que afrontan las colecciones antropológicas se deben principalmente a que los ejemplares están compuestos por sustancias proteicas, que componen los huesos, pelos y la piel así como de materiales celulósicos en sus elementos accesorios; sustratos potenciales para el desarrollo de agentes de biodeterioro como los hongos (Saparrat et al., 2010). La disponibilidad de éstos últimos como esporas en el ambiente circundante, incluyendo sobre soportes de yeso, arcilla, madera, vidrio, papel y/o plástico, puede incidir en la preservación del ejemplar conservado puesto que las condiciones de temperatura y humedad relativa así como las características de edificación del sitio de almacenamiento determinan su germinación y activación en el ataque de los materiales de valor. También se pueden detectar adicionales efectos negativos vinculados con otras alteraciones sobre las propiedades físico-químicas y mecánicas del material conservador y su soporte de exhibición (Pinheiro \& Filomena, 2009; Sterflinger 2010),

A todo esto hay que añadir las modificaciones del aspecto estético del material en cuanto a alteraciones cromáticas y de superficie que acrecientan el proceso de biodeterioro. La intensidad de las transformaciones, se produce en función de los componentes de los soportes y de las condiciones ambientales (Mesquita et al., 2009; Valentín et al., 2010).

El origen de las colecciones, pertenecientes a la División Antropología del Museo de la Plata, es el producto de las investigaciones de los primeros naturalistas que comenzaron a explorar el territorio argentino a finales del siglo XIX. Dentro de este grupo se destacó Francisco P. Moreno, quien en 1877 donó su colección con el fin de fundar el Museo de Antropología y Arqueología. En el año 2006, las autoridades del Museo de La Plata decidieron retirar de exhibición los restos humanos que no contaban con el consentimiento de su comunidad de pertenencia (http/:www.museo.com.ar). Esta decisión fue motivada por pedidos de los pueblos originarios, a través de la sanción de la ley nacional 25.517 y las recomendaciones internacionales de ética para museos (http/: www.icom.org).

Teniendo en cuenta que muchos de estos restos pertenecieron a personas identificadas y que se hallan esperando que se cumpla con todos los procesos legales para su restitución, y en el marco del respeto indispensable a su identidad cultural se acondicionó en el subsuelo del museo un sitio de Guarda en la ex Sala Ameghino (Fig. 1, A) en la cual se llevan a cabo periódicamente ceremonias de homenaje a miembros de comunidades indígenas cuyos restos se encuentran en dicha Sala. Los restos humanos conservados proceden de diversos sitios arqueológicos de Argentina, Bolivia y Chile depositados en cajas o en vitrinas con materiales especiales para su soporte y conservación. Diez de ellos proceden de la localidad de Pampa Grande Departamento de Guachipas, Serranías de las Pirguas (Provincia de Salta) datados de 500-600 d.C; algunos de los cuales se ubican en contenedores especiales según características particulares que permiten conservar la posición original y ajuares funerarios tal como lo presentaban en exhibición (Reca et al., 2009).

El objetivo del presente trabajo fue llevar a cabo un relevamiento de las condiciones microbiológicas de la Sala para garantizar la mejor conservación de los restos humanos en guarda hasta el momento en el que se concrete la Restitución. Para ello se analizó la carga fúngica ambiental y otros bioaerosoles asociados al depósito mencionado. Para ello se llevaron realizaron dos tipos de monitoreos, i) uno del tipo aerobiológico en el aire exterior del depósito (ex Sala Ameghino) del subsuelo del Museo, en el aire interior del mismo y en el interior de 3 de sus vitrinas donde se hallan restos humanos; y ii) otro que consistió en el estudio de la micobiota asociada a la superficie de materiales seleccionados con signos evidentes de deterioro. 
D. S. Nitiu et al. - Carga fúngica en depósito con restos humanos en MLP
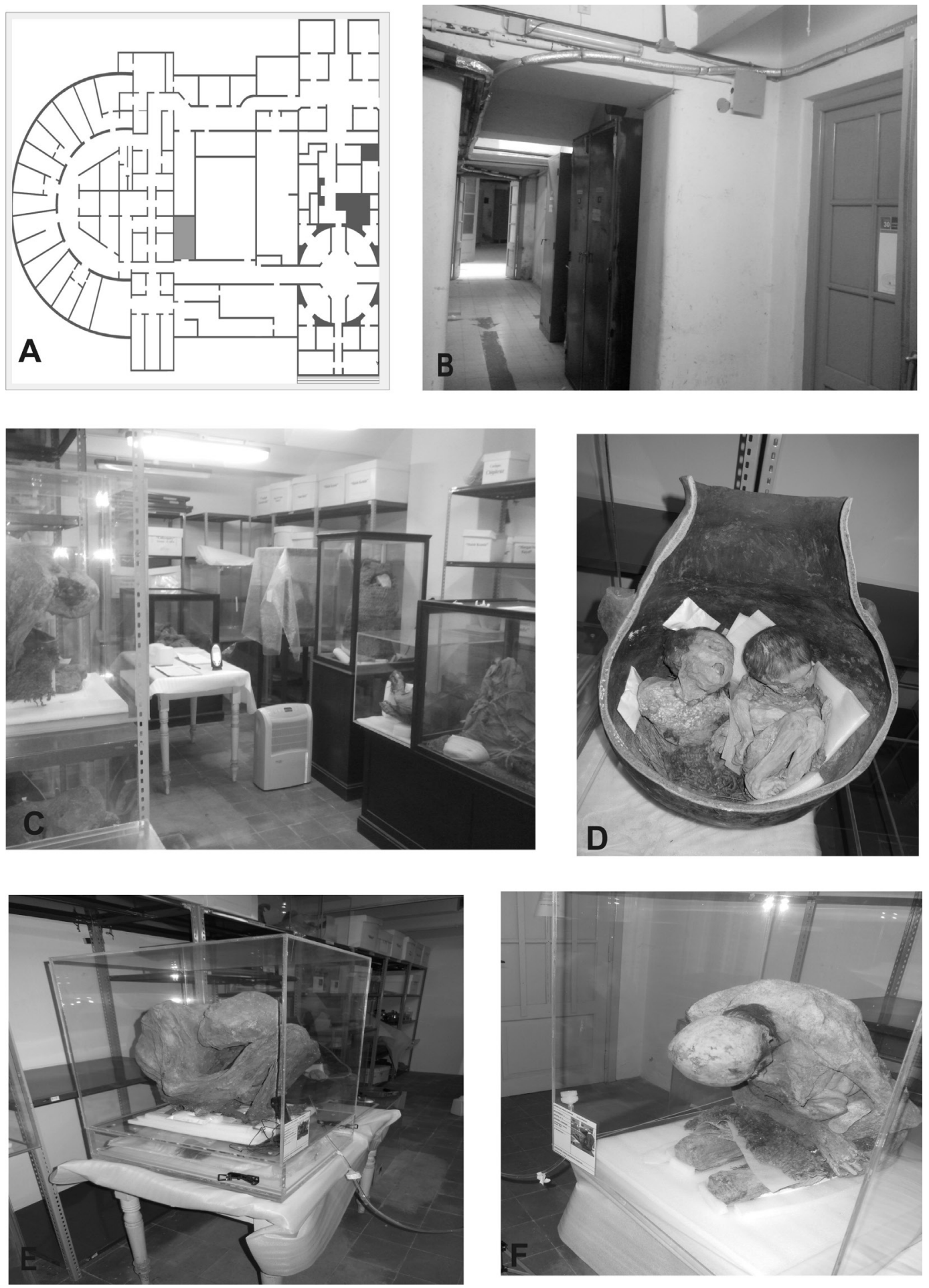

Fig.1. A: Plano del ala izquierda del subsuelo del Museo de LP. Área gris corresponde al Depósito ex Aula Ameghino. B: Pasillo exterior al depósito. C: Recinto donde se custodian los restos humanos momificados. D: Vitrina I de estudio. E: Vitrina II. F: Vitrina III. 


\section{Materiales Y MÉtodos}

\section{Sitio de muestreo}

El Depósito (ex Sala Ameghino) está ubicado en el subsuelo del Museo de Ciencias Naturales de La Plata y posee una superficie de $28 \mathrm{~m}^{2}$ con 7 vitrinas y numerosas estanterías que alojan material antropológico al que se accede por una entrada que da a un pasillo interior del Museo (Fig. 1, B). La única iluminación del depósito es artificial y circunstancial por acceso de personal. Para mantener constante las condiciones ambientales del depósito $\left(26^{\circ} \mathrm{C}\right.$ y $50 \%$ de humedad relativa) y así minimizar el deterioro del material conservado, el sitio cuenta con un aparato de aire acondicionado y un deshumidificador. La temperatura y la humedad relativa son registrados a diario por personal técnico utilizando un termohigrómetro data logger HOBO U14 LCD en el centro del depósito. (Fig. 1, C). Asimismo, cada vitrina también contiene en su interior otro termohigrómetro equivalente que registra la temperatura y humedad relativa. Esta información provee continuamente las condiciones microambientales de cada sitio con la finalidad de evitar variaciones que puedan generar fracturas $u$ otras modificaciones en el material momificado y así contribuir al control de la estabilidad ambiental.

Se seleccionaron para este estudio 3 vitrinas representativas del depósito en cuyo interior se hallan restos momificados de la localidad de Pampa Grande, Departamento de Guachipas, Serranías de las Pirguas (Provincia de Salta):

Vitrina I: contiene los cuerpos de dos niños sentados en el interior de una vasija de cerámica, acompañados de restos de telas y mimbrería (Fig. 1, D);

Vitrina II: es el reservorio de un cadáver adulto naturalmente momificado en posición fetal y genuflexa que presenta evidentes signos de deterioro como pequeños orificios en las partes óseas y manchas blancas en la superficie de todo el cuerpo (Fig. 1, E); y

Vitrina III: alberga un cadáver adulto naturalmente momificado en posición fetal y arrodillado, acompañado por un ajuar conformado por textiles de lana y fibra (probablemente pertenecientes al fardo funerario que lo envolvía) (Fig. 1, F).

\section{Muestreos}

Toma de muestras aerobiológicas

Para el muestreo del aire del ambiente exterior e interior del depósito así como del interior de cada una de las tres vitrinas, se utilizaron dos métodos volumétricos, uno no-viable, a través de la observación directa de la muestra al microscopio óptico, y otro viable, basado en el cultivo de la suspensión resultante sobre un medio estándar para el crecimiento de hongos ambientales.

Los muestreos aerobiológicos no-viables se llevaron a cabo a través de la toma de muestras de aire con una bomba aspirante Z-LiteIAQ Pump calibrada en $15 \mathrm{l} / \mathrm{m}$ conectada a un cassette Air o Cell descartable, siendo cada muestra el resultado de las partículas capturadas en el transcurso del pasaje del aire durante 5 minutos. Cada muestra cassette obtenida se procesó siguiendo el protocolo de Air O Cell ${ }^{\circledR}$ siendo montada y teñida con azul de algodónlacto-fenol para su análisis. Este último consistió en la observación de la muestra en 7 transectas transversales al eje principal del preparado a un aumento de $400 \mathrm{X}$ en el microscopio óptico, el cual representa el $22 \%$ del total muestreado. Se identificaron los bioaerosoles, con énfasis en las esporas fúngicas, utilizando atlas de referencia (Käärik et al., 1983; Barnet \& Hunter, 1987; Grant Smith, 1990; Lacey \& West, 2006) y bases de datos especializadas.

Paralelamente se analizó también la micobiota aérea viable (cultivable) utilizando un portafiltro Millipore equipado con un papel de filtro estéril (en reemplazo del cassette Air o Cell descartable) conectado a la mencionada bomba aspirante, siendo también expuesto por 5 minutos a la corriente del aire a un flujo de $15 \mathrm{l} / \mathrm{m}$. Los filtros correspondientes a cada muestra se trituraron y homogeneizaron en 100 $\mathrm{ml}$ de agua destilada estéril obteniendo una suspensión utilizada como fuente de inóculo. Un mililitro de alícuotas decimales seriadas de cada suspensión de partida se sembraron en placas conteniendo el medio agar-harina de maíz-glucosa (Cabello \& Arambarri, 2002) suplementado con sulfato de estreptomicina $(0,5 \%)$ y cloramfenicol $(0,25 \%)$. Se realizaron tres réplicas de siembra por dilución de suspensión analizada. Las placas se incubaron a $27^{\circ} \mathrm{C}$ hasta desarrollo de colonias, las cuales fueron identificadas taxonómicamente y cuantificadas. La identificación de los taxa fúngicos se realizó utilizando bibliografía de referencia (Barron, 1968; Carmichael et al., 1980; Domsch et al., 1993; Ellis, 1971, 1976).

\section{Toma de muestras de superficie}

Con la finalidad de identificar la micobiota asociada 
al deterioro de materiales específicos de las momias presentes en las vitrinas, se procedió a la toma de muestras de superficie en zonas con signos evidentes de deterioro mediante la aplicación de hisopos estériles (contacto) y pinzas (obtención de tejidos). Fragmentos de los hisopos utilizados y de los tejidos obtenidos, individualmente conservados en tubos estériles, se sembraron sobre los medios agar-extracto de papa-glucosa (APG), agar-harina de maíz y agarextracto de malta suplementados con estreptomicina y cloramfenicol en las proporciones arriba indicadas, siendo las placas incubadas a $27^{\circ} \mathrm{C}$ hasta desarrollo de colonias. Las colonias fúngicas desarrolladas se identificaron taxonómicamente siguiendo la metodología reportada por Elíades et al., (2014).

Además se llevó a cabo un muestreo de contacto utilizando portaobjetos siliconados especiales que luego de su exposición fueron observados al microscopio óptico.

\section{Análisis de datos}

Acorde a un estudio de caso, la toma de muestra en cada sitio consistió sólo en una muestra representativa del análisis no-viable (sistema cassette) y otra del viable (sistema portafiltro) con tres réplicas. La concentración de los diferentes tipos esporales identificados se estimó siguiendo el procedimiento de Baxter (2006) sobre el recuento derivado de las muestras no-viables y se expresó en base a concentración de esporas /metro cúbico de aire. La carga fúngica viable se estimó sobre la base del recuento de colonias fúngicas totales por dilución y se expresó en $\mathrm{UFC} / \mathrm{m}^{3}$.

\section{Resultados}

El muestreo se llevó a cabo el día 22 de noviembre de 2011 entre las 10 y $12 \mathrm{am}$, en ese lapso, la temperatura del sitio era de $26^{\circ} \mathrm{C}$ y la humedad de $50 \%$ mientras que la temperatura exterior al recinto era de $28^{\circ} \mathrm{C}$ y la humedad de $70 \%$.

\section{Pasillo exterior}

Con fines comparativos se realizó el análisis aerobiológico del pasillo exterior al depósito el cual permitió identificar 8 morfotipos según la metodología no-viable cuantificando un promedio de 313 esporas $/ \mathrm{m}^{3}$ y 7 géneros esporales según metodología viable, estimándose un promedio de 67
$\mathrm{UFC} / \mathrm{m}^{3}$. Cladosporium herbarum fue el hongo más frecuente mientras que Cladosporium cladosporioides, Alternaria sp. y Penicillium sp. fueron los taxa comunes en ambos muestreos (Tabla 1).

Asimismo se detectaron fragmentos orgánicos, tipo escamas de piel, probablemente debido a que es un sitio de tránsito de personas y con conexión con otras áreas visitadas del museo. Se identificaron otras partículas opacas asociadas al polvo ambiental, aunque estás no se pudieron asignar a ningún foco de origen fúngico.

\section{Recinto}

En base al muestreo para recuento no-viable en el aire del recinto del depósito se encontraron los siguientes morfotipos esporales: Alternaria sp., Cladosporium cladosporioides y Coprinus sp., alcanzando un valor promedio de 99,36 esporas $/ \mathrm{m}^{3}$ (Tabla 2). Se detectó también material particulado como escamas epidérmicas (fragmentos de piel o caspa), fibras hialinas (pudiendo corresponder a partículas biogénicas como fibras celulósicas o sintéticas) y algunas partículas opacas derivadas de construcción. Con la muestra tomada en el mismo aire ambiental a partir del sistema para cultivo se identificaron 5 taxa fúngicos con un valor promedio de 54,6 UFC $/ \mathrm{m}^{3}$ siendo Penicillium frequentans el tipo más frecuente en las réplicas.

\section{Vitrinas}

Según la metodología de observación directa, en el aire interior de la Vitrina I, se identificaron

Tabla 1. Diversidad fúngica identificada en el

pasillo exterior del depósito, comparando las dos metodologías de muestreo empleadas.

\begin{tabular}{|ll|}
\hline \multicolumn{1}{|c|}{ METODO NO VIABLE } & \multicolumn{1}{c|}{ METODO VIABLE } \\
Tlternaria sp. & \multicolumn{1}{c|}{ Taxa } \\
Arthrinium $\mathrm{sp}$. & Alternaria alternata \\
Aspergillus-Penicillium & $\begin{array}{l}\text { Cladosporium } \\
\text { cladosporioides } \\
\text { Cladosporium } \\
\text { herbarum }\end{array}$ \\
Chaetomium sp. & Penicillium frequentans \\
Cladosporium cladosporioides & Penicillium rubrum \\
Epicoccum $\mathrm{sp}$. & Penicillium thomii \\
Leptosphaeria type & Rhodotorula sp. \\
Myxomycota & Micelio blanco \\
\hline
\end{tabular}


Tabla 2. Diversidad fúngica identificada en el interior del depósito.

\begin{tabular}{|c|c|}
\hline $\begin{array}{l}\text { METODO NO VIABLE } \\
\text { por cassette }\end{array}$ & $\begin{array}{l}\text { METODO VIABLE } \\
\text { por filtro }\end{array}$ \\
\hline Tipo morfológico & Taxa \\
\hline Coprinus sp. & Aspergillus niger \\
\hline Alternaria sp. & Purpureocillium lilacinum \\
\hline \multirow[t]{3}{*}{ Cladosporium cladosporioides } & Penicillium frequentans \\
\hline & Penicillium rubrum \\
\hline & Talaromyces sp. \\
\hline
\end{tabular}

los morfotipos Alternaria sp., Cladosporium cladosporioides y Coprinus sp., (Tabla 3) así como partículas opacas; estas últimas posiblemente derivadas del propio material momificado. El análisis viable reveló en el interior de la mencionada vitrina una carga fúngica de $48,3 \mathrm{UFC} / \mathrm{m}^{3}$ correspondiente a 6 taxa, con Penicillium frequentans como el más frecuente. La inoculación de las muestras tomadas con pinzas del pecho, la espalda y el pelo del cuerpo momificado evidenciaron 4 taxa fúngicos asociados a estos sustratos (Tabla 3).

El monitoreo a través del sistema no viable del aire interior de la Vitrina II mostró únicamente al morfotipo Torula sp. con un total de 59,62 propágulos/ $\mathrm{m}^{3} \mathrm{y}$ en asociación a la presencia de partículas opacas (Tabla 3). Utilizando el sistema in-vitro se estimaron $47 \mathrm{UFC} / \mathrm{m}^{3}$ y se recuperaron los taxa Aspergillus niger, Penicillium rubrum y Penicillium frequentans. Asimismo empleando secciones de tejidos asociados a la cadera, pierna y abdomen seleccionados para su inoculación se detectó la presencia de hongos correspondientes a 5 taxa, incluyendo a Cladosporium herbarum.

A partir de la observación directa de la muestra tomada en el aire interior de la Vitrina III se encontró al tipo morfológico Alternaria sp. con un total de 119,24 esporas $/ \mathrm{m}^{3}$, siendo acompañado por partículas opacas y fibras hialinas. El muestreo del aire de la mencionada vitrina con el sistema viable reveló un valor promedio de $45 \mathrm{UFC} / \mathrm{m}^{3}$, siendo representado por 3 taxa fúngicos (Tabla 3). Sobre el medio APG, fragmentos del cuerpo momificado presente en la vitrina resultaron ser fuente de inóculo de Penicillium frequentans y Talaromyces helicus (teleomorfo de Penicillium sp.).

No se detectaron propágulos fúngicos en los portaobjetos siliconados expuestos al contacto de la superficie de los cuerpos momificados.

\section{Discusión y Conclusiones}

La presencia de estructuras fúngicas y su carga en sitios donde se custodian colecciones biológicas o piezas de importancia histórica representan una potencial amenaza tanto para el material como para el personal involucrado dado que muchos de los hongos del aire en especial Asperguillus sp., Penicillium sp. y Cladosporium están considerados como alérgenos primarios pudiendo causar diversos problemas respiratorios en los humanos (Jarvis \& Millar, 2005; Genuis, 2007).

En la actualidad no existe un consenso internacional que permita clasificar a partir de que concentración se puede considerar un ambiente interior con "contaminación fúngica", aunque hay diversas normativas. La norma dispuesta por la Conferencia Americana de Higienistas Industriales Gubernamentales (ACGIH) y el US Public Health Service establecen un umbral de $200 \mathrm{UFC} / \mathrm{m}^{3}$ para bioaerosoles fúngicos. Por otra parte, la Unión Europea establece en las normas técnicas de medición de Baubiologie (Standard of Building Biology Tesing Methods and Guidelines, SBM), $500 \mathrm{UFC} / \mathrm{m}^{3}$ como límite máximo que infiere una alta contaminación. Mientras tanto, instituciones como la Healthy Building International y Brazilian Healthy Ministry establecen $750 \mathrm{UFC} / \mathrm{m}^{3}$ como umbral y autores como Reponen et al., (1990) consideran $300 \mathrm{UFC} / \mathrm{m}^{3}$.

Analizandolos datos del presente trabajo, el pasillo exterior al depósito fue el sitio donde se cuantificó un mayor número de esporas a través de ambos métodos. Puesto que los niveles de concentración no superaron los valores límites establecidos por alguna de las normativas internacionales mencionadas, la carga aeromicológica disponible en el depósito antropológico y su ambiente exterior 
Tabla 3. Morfotipos fúngicos identificados en Vitrina I, Vitrina II y Vitrina III según método no viable por medio de cassette y taxa fúngicos determinados a través de método viable por medio por medio de filtros

y por el uso de pinzas.

\begin{tabular}{|c|c|c|c|}
\hline \multicolumn{4}{|l|}{ VITRINA 1} \\
\hline \multirow{2}{*}{$\begin{array}{l}\text { METODO NO VIABLE } \\
\text { por cassette } \\
\text { Tipo morfológico }\end{array}$} & \multicolumn{3}{|c|}{ METODO VIABLE } \\
\hline & por filtro & & $\begin{array}{l}\text { por pinzas } \\
\text { Taxa }\end{array}$ \\
\hline Coprinus sp. & Rhodotorula sp. & Pecho & Beauveria bassiana \\
\hline Alternaria sp. & Baeuveria bassiana & Pelo & Fusarium aff. oxysporum \\
\hline \multirow[t]{4}{*}{ Cladosporium cladosporioides } & Penicillium frequentans & Espalda & Purpureocillium lilacinum \\
\hline & Penicillium rubrum & & Cladosporium herbarum \\
\hline & Epiccocum nigrum & & \\
\hline & Talaromyces sp. & & \\
\hline \multicolumn{4}{|l|}{ VITRINA 2} \\
\hline \multirow[t]{6}{*}{ Torula sp. } & Aspergillus niger & Abdomen & Cladosporium herbarum \\
\hline & Penicillium frequentans & & Alternaria alternata \\
\hline & Penicillium rubrum & Cadera & Rhodotorula sp. \\
\hline & & Piernas & Cladosporium herbarum \\
\hline & & & Fusarium aff. oxysporum \\
\hline & & & Purpureocillium lilacinum \\
\hline \multicolumn{4}{|l|}{ VITRINA 3} \\
\hline \multirow[t]{4}{*}{ Alternaria sp. } & Talaromyces sp. & Cuerpo & Talaromyces sp. \\
\hline & Penicillium frequentans & & Penicillium frequentans \\
\hline & Penicillium rubrum & & Epiccocum nigrum \\
\hline & & & Cladosporium sp. \\
\hline
\end{tabular}

inmediato no parece representar un sitio con contaminación fúngica.

Asimismo, es de destacar el posible efecto de factores ambientales como la corriente de aire, la cual puede jugar un papel clave en lo que se refiere a la limpieza de la nube de polvo y demás partículas existentes en la zona de estudio (Borrego \& Perdomo, 2014). Sin embargo, el pasillo es vulnerable a los cambios de temperatura y humedad relativa a lo largo del año, lo que puede favorecer a la presencia de diversos anamorfos de Ascomycota acordes a su disponibilidad y arribo desde otras áreas exteriores y su desarrollo y multiplicación sobre soportes y materiales accesibles.

El aire exterior entra en la sala únicamente por medio del reducido transito del personal autorizado para su aseo, monitoreo y estudio que accede a la misma, cuya función, entre otras cotidianas, es controlar a diario la temperatura y humedad relativa a través del registro del aire acondicionado y del deshumidificador. Las ventanas fueron selladas y la puerta reacondicionada, tratando de minimizar los cambios bruscos en el ambiente. En condiciones ambientales apropiadas, la micobiota puede coexistir latente con las colecciones en estado de esporas; sin embargo la existencia de propágulos de géneros fúngicos en el aire de estos recintos puede incidir en el desarrollo de enfermedades respiratorias lográndose asociar la presencia de los mismos con el desarrollo de síntomas consistentes con este tipo de patologías (Nevalainen \& Morawska, 2010; Raphoz et al., 
2010; Albright 2001). Asimismo, un aumento en los registros de temperatura y humedad relativa puede conducir a activar los propágulos y su germinación y desarrollo miceliar puede hacer estragos en las colecciones acelerando su biodeterioro (Pinzari et al., 2004).

Se evidenció en el ambiente del depósito de momias como en los interiores de las vitrinas la presencia y predominio de Penicillium sp., Cladosporium sp. y Aspergillus sp.. Estos taxa fúngicos han sido reportados por Singh (2003), Florian (2003), Valentin (2010) y Solis (2011) como los tres principalesgéneros contaminantes de ambientes interiores a nivel mundial y comúnmente aislados en casas, archivos, bibliotecas y museos (Walter, 2003) (Gutarowska, 2010), cuyo desarrollo puede estar incluso favorecido por la situación ambiental controlada del depósito.

De acuerdo con Icenhour \& Levetin (1997) y Calderón et al., (1997), es común encontrar a estos hongos mitospóricos en ambientes secos dado que al aumentar la temperatura las esporas sufren desecación y así se facilita su dispersión en el aire. Asimismo, la limpieza minuciosa del material con equipamiento sofisticado puede también resuspender la carga espórica adsorbida a los materiales y así aumentar su disponibilidad en el ambiente.

El aire sirve primariamente como un medio de transporte para los hongos y sus diásporas, no siendo fuente de nutrientes o condiciones (hábitat) que propicien su acción en el biodeterioro. El espectro de dichos propágulos depende de la colonización natural del ambiente, de su viabilidad y de la transmisión y procesos de transformación (Lighthart \& Stetzenbach, 1994). La concentración de los mismos depende en gran medida de varios factores tales como la temperatura y la humedad relativa, el movimiento del aire, la luz y las fuentes de inóculo. Por ejemplo, la luz ultravioleta inhibe el crecimiento y la ausencia de luz impide la formación de esporas de algunos hongos como Alternaria sp. (Diaz Rojas et al., 2010).

El desarrollo de los microorganismos, incluido los hongos ambientales, se detiene cuando la actividad del agua soporte es menor de la que necesita. Ello no implica la muerte del microorganismo, sino que éste se mantiene en una fase resistente durante un período de tiempo variable (García Miniet \& Sánchez Espinosa, 2012). En el caso de las esporas, la fase de resistencia puede prolongarse durante años no interpretándose como alarmantes para el desarrollo de biodeterioro (Florian, 2004). Los hongos en estado vegetativo, ya sea en la forma de micelio y/o unicelular, presentan una actividad fisiológica clave en el proceso de la degradación de diversos soportes utilizados como sustratos de crecimiento (Guiamet et al., 2006; Cappitelli \& Sorlini, 2010).

El uso de muestreos volumétricos no-viable y viable permite cubrir un espectro mayor de la diversidad de taxa particulares en cada caso, ya sea por las características propias de cada espora, su frecuencia, o por las condiciones favorables o limitantes para su detección. Aunque los microorganismos cultivables pueden representar solo una pequeña fracción de los recuentos viables y no-viables, los procedimientos que utilizan métodos de cultivo siguen siendo la técnica más ampliamente utilizada para la recolección e identificación de hongos y bacterias ambientales (Parat et al., 1999). Según la metodología no viable se identificaron 11 tipos esporales exclusivos pertenecientes a los phyla Ascomycota (Arthrinium sp., Chaetomium sp. Tipo Leptosphaeria sp. y Torula sp.) y Basidiomycota (Coprinus sp.) así como también a representantes morfológicamente afines Tipo Myxomycota (phylum Mycetozoa). Mientras que según metodología viable se identificaron 12 géneros esporales exclusivos siendo mayoritariamente del phylum Ascomycota (Beauveria sp., C. herbarum, Fusarium sp., Purpureocillium sp. y Talaromyces sp.), aunque también se detectó un representante del phylum Basidiomycota (Rhodotorula sp.). La combinación de ambas metodologías resulta efectiva para una mayor recuperación de la diversidad de elementos presentes en el aire dado que el sistema de observación directa permite identificar además de esporas y propágulos fúngicos, otras partículas biológicas presentes en el aire como escamas, pelos y partículas no biológicas u opacas. Y por otro lado, el sistema de captura para aislamiento y cultivo invitro permite realizar determinaciones sistemáticas de formas cuyas esporas tienen morfologías no diagnósticas y diferenciales entre géneros que si son fácilmente identificables por su ontogenia conidial.

Estudios adicionales involucrando herramientas moleculares pueden ser de utilidad en la 
identificación de hongos no cultivables en medios micológicos de rutina y/o poco frecuentes que incluso estén también asociados al depósito bajo estudio. Se recomienda monitorear la micobiota con frecuencia tanto la del aire de los ambientes como del material patrimonial de depósitos de museos puesto que permite garantizar una referencia del sitio como así también, proporcionar información acerca de aquellos tipos fúngicos representativos del aire con destacada habilidad para modificar, degradar y por lo tanto con potencial para deteriorar el material de las momias y la consecuente pérdida estética de ellas. Dada la importancia cultural que tienen los cuerpos momificados del NOA, hoy en custodia en el Museo de Ciencias Naturales de La Plata, la detección temprana de agentes con posible actividad negativa sobre los mismos es prioritaria dado que pueden provocar serios problemas de deterioro y así repercutir en la conservación de este patrimonio invalorable de la antropología regional.

\section{Agradecimientos}

Los autores agradecen el apoyo financiero concedido por el Consejo Nacional de Investigaciones Científicas y Tecnológicas (CONICET) PIP 112201101-00087 y PIP 112-201101-00391 y Agencia Foncyt PICT 2013-0418 y PICT 2011-0501. Un especial agradecimiento a la Coordinadora María Marta Reca, Directora de la Unidad de Conservación y Exhibición del Museo de La Pata (UCE) y a la Dra. M.A. Morbelli por su apoyo incondicional.

Daniela S. Nitiu, Lorena A. Elíades y Mario C. N. Saparrat son investigadores del CONICET. Andrea C. Mallo es personal de apoyo de CIC (Provincia de Buenos Aires).

\section{Bibliografía}

ALBRIGHT, D. M. 2001. Human health effects of airborne mycotoxins exposure in fungi-contaminated indoor environment. Professional Safety: 26-28.

BARNET, H. L. \& B. B. HUNTER. 1987. Illustrated Genera of Imperfect Fungi. MacMillan Publ. Co. New York.

BARRON, G. L. 1968. The Genera of Hyphomycetes from Soil, Williams \& Wilkins, Baltimore.

BAXTER, A. 2006. Air O Cell Interpretation guide. Disponible en: http: https://www.ehs.umass.edu/air- o-cell-method-interpretation-guide. [Acceso: Enero 2011]

BORREGO, S. \& I. PERDOMO AMISTAD. 2014. Caracterización de la micobiota aérea en dos depósitos del Archivo Nacional de la República de Cuba. Rev Iberoam Micol. 31: 182-187.

CABELLO, M. N. \& A. M. ARAMBARRI. 2002. Diversity in soil fungi from undisturbed and disturbed Celtis tala and Scutia buxifolia forests in the eastern Buenos Aires province (Argentina). Microbiol. Res. 157: 115-125.

CALDERON, C., J. LACEY, A. Mc. CARTNEY \& I. ROSAS. 1997. Influence of urban climate upon distribution of airborne Deuteromycete spore concentration in México City. Int. J. Biometeorol 40: 71-80.

CAPPITELLI, F. \& C. SORLINI. 2010. Paper and manuscripts. In MITCHELL R. MCNAMARA Cl. Eds. Cultural Heritage microbiology: Studies in conservation science. ASM Press. 45-58. Washington. DC.

CHARMICHAEL, J. W., W. BRYCE KENDRICK, I. L. CONNERS \& L. SIGLER. 1980. Genera of Hyphomycetes. The University of Alberta Press, Edmonton.

DÍAZ ROJAS, M., J. GUTIÉRREZ ESPINOSA, A. GUTIÉRREZ ESPINOSA, MdC GONZÁLEZ CHÁVEZ, G. VIDAL GAONA, R. M. ZARAGOZA PALENCIA \& C. CALDERÓN EZQUERRO. 2010. Caracterización aerobiológica de ambientes intramuro en presencia de cubiertas vegetales Rev. Int. Contam. Ambient 26 no.4 México

DOMSCH, K. H, W. GAMS \& T. ANDERSON. 1993. Compendium of soil fungi. IHW-Verlag, Eching.

ELÍADES, L., M. N. CABELlO, V. PANCOTTO, A. MORETTO, M. M. RAGO \& M. C. N. SAPARRAT. 2014. Preliminar data on growth and enzyme abilities of soil fungus Humicolopsis cephalosporioides under different incubation temperatures. Rev. Iberoam. Micol. 32: 40-45.

ELLIS, M. B. 1971. Dematiaceous Hyphomycetes. Ed. Commonwealth Mycological Institute. Kew.

ELLIS, M. B. 1976. More Dematiaceous Hyphomycetes. Ed. Commonwealth Mycological Institute. Kew.

FLORIAN M. L. E. 2003.

GARCIA MINIET, M. \& R. SÁNCHEZ ESPINOSA. 2012. Estudio de la concentración fúngica aérea de los depósitos del Archivo Municipal de Cárdenas, Cuba. Revista de la Sociedad Venezolana de Microbiología 32: $37-43$

GENUIS, S. J. 2007. Clinical medicine and the budding science of indoor mold exposure. Eur J. Int. Med. 18: 516-523.

GUTAROWSKA, B. 2010. Metabolic activity of moulds as a factor of building materials biodegradation. Polish $J$. Microbiol 59: 119-124. 
GRANT SMITH, E. 1990. Sampling and identifying allergenic pollens and molds. Blewstone Press, San Antonio.

GUIAMET, P. S., S.G. GOMEZ DE SARAVIA, P. ARENAS, M. L. PEREZ, J. DE LA PAZ, S. F. BORREGO. 2006. Natural products isolated from plant used in biodeterioration control. Pharmacology Online 3: 537-544.

HARVEY, 1977. La política cultural en Argentina. UNESCO. ISBN: 92.301547-5

ICENHOUR, C. R. \& E. LEVETIN. 1997. Penicillium and Aspergillus species in the habitats of allergy patients in the tulsa, Oklahoma area. Aerobologia 13: 161-166.

ICOM. Código de ética Profesional de los Museos. 1946. http://www.icom.org/

JARVIS, B. B. \& J. D. MILLER. 2005. Mycotoxins as harmful indoor air contaminants. Appl. Microbiol Biotechnol 66: 367-372.

KÄÄRIK, A, J. KELLLER, E. KIFFER, J. PERREAU \& O. REISINGER.1983. Atlas of airborne fungal spores in Europe. Springer-Verlag, Berlin.

LACEY, M. E. \& J. S. WEST. 2006. The Air Spore. Springer, Dordrecht.

LIGHTHART, B. \& L. D. STETZENBACH. 1994. Distribution of microbial bioaerosol. In: LIGHTHART B. \& MOHR A. J. (eds.), Atmospheric Microbial Aerosols. Theory and Applications. Chapman and Hall. New York. London. 68-98.

MESQUITA N., A. PORTUGAL, S. VIDEIRA, S. RODRIGUEZ-ECHEVERRÍA, A. M. L BANDEIRA., M. J. A. SANTOS \& H. FREITAS. 2009. Fungal diversity in ancient documents. A case study at the Archive of the University of Coimbra. Int. Biodeterior. Biodegrad, 63: 626-629.

NEVALAINEN A. \& MORAWSKA L. 2010. Biological agents in indoor environments. Assessment of health risks. Work conducted by a WHO Expert Group between 2000-2003. http://www.ilaqh.qut.edu. au/ Misc/BIOLOGICAL_AGENTS_2009.pdf.

PARAT, S., .PERDRIX A., MANN S., .BACONNIER P. 1999. Contribution of particle counting in assessment of exposure to airborne microorganisms. Atmospheric Environment. 33: 951-959.

PINHEIRO A. \& FILOMENA M. 2009. Risk assessment a comparative study of archive storage rooms. Journal of Cultural Heritage. 10: 428-434.

PINZARI F, C.FANELLI, O.CANHOTO \& N. MAGAN 2004. Electronic nose for the early detection of moulds in libraries and archives. Indoor Built Environment, 13: 387- 395.

RAPHOZ M., M.S.GOLDBERG, M.GARNEAU, L.HËGUY, M.F. VALOIS \& F. GUAY 2010. Associations between atmospheric concentrations of spores and emergency department visits for asthma among children living in Montreal. Archives of Environmental \& Occupational Health 65: 201-210.

RECA M. M., B. B. HARA, S. E. MARCIANESI, \& S. DE LA CRUZ. 2009. Proyecto integral de acondicionamiento de cuerpos momificados de la Colección del Museo de La Plata. Revista Argentina de Antropología Biológica 11: 143-155.

REPONEN, T., A. NEVALAINEN, M. JANTUNEN, M. PELLIKKA \& P. KALLIOKOSKI. 1990. Normal range criteria for indoor air bacterial and fungal spores in a subantartic climate. Indoor air 2: 26-32.

SAPARRAT, M. C. N., J.M. ESTEVEZ, M. I. TRONCOSO, A. M. ARAMBARRI \& P. A. BALATTI. 2010. Invitro depolymerization of Scutia buxifolia leaf-litter by a dominant Ascomycota Ciliochorella sp. Inter. Biodeterior. Biodegrad. 64: 262-266.

SINGH, J. 2003. European Heritage conservation and environmental monitoring-making informed decisions. European Heritage conservation environmentally sustainable Solutions. Dublin, Ireland, Trinity College.

SOLÍS, E. 2011. Estudio micológico del aire en áreas ocupacionales y exteriores del laboratorio de investigación en productos naturales ubicado en el edificio T-10 en la ciudad universitaria en zona 12 y el laboratorio ubicado en la zona 1 del centro de información y asesoría toxicológica del departamento de toxicología de la facultad de ciencias químicas farmacia de la universidad de San Carlos de Guatemala. 2011 [On líne]. Disponible en web: biblioteca.usac.edu.gt/tesis/06/06_3102.pdf. [Acceso: 6 de jun. 2012].

STERFLINGER, K. 2010. Fungi: their role in deterioration of cultural heritage. Fung. Biol. Rev. 24: 47-55

VALENTIN, N. 2010. Microorganism in museum collections. Coalition. $19: 2-5$.

VALENTIN, N., C. MURO \& J. MONTERO. 2010. Métodos y Técnicas para Evaluar la Calidad del Aire en Museo: Museo Nacional Centro de Arte Reina Sofía”. Ed. CARS - IIC Grupo Español: 63-81.

WALTER, A. 2003. Basic preservation guidelines for library and archive collections. London: Natural Preservation Office, NPO Preservation Ghidance. British Library.

Recibido el 17 de abril de 2015, aceptado el 9 de septiembre de 2015. 